\author{
* Presented in part at the 36th Meeting of the Israel Chemical \\ Society, Ramat-Aviv, Israel. \\ ${ }^{1}$ J. Padova, J. Chem. Phys. 39, 1552 (1963). \\ 2 B. E. Conway, Ann. Rev. Phys. Chem. 14, 481 (1966). \\ ${ }^{3}$ J. Padova, Electrochim. Acta 12, 1227 (1967). \\ 4 W. A. Millen and D. W. Watts, J. Am. Chem. Soc. 89, 6051 \\ (1967). \\ 6 H. S. Frank, J. Chem. Phys. 23, 2023 (1955). \\ ${ }^{6}$ J. Padova, J. Phys. Chem. 42, 796 (1968). \\ 7 C. Grahame, J. Chem. Phys. 21, 1054 (1953). \\ 8 (a) J. Malsch, Ann. Phys. 84, 841 (1927); Phys. Z. 29, 770 \\ (1928); P. S. Rao and D. P. Swarup, J. Chem. Phys. 43, 4530 \\ (1965). (b) The author is indebted to Dr. M. Gazith for his \\ assistance in running this program. \\ H. S. Frank and W. Y. Wen, Discussions Faraday Soc. 24, \\ 133 (1957). \\ ${ }^{10}$ O. Redlich and D. M. Meyer, Chem. Rev. 64, 221 (1964).
}

${ }^{11} \mathrm{~J}$. Padova and I. Abrahamer, J. Phys. Chem. 71, 2112 (1967).

${ }^{12}$ B. E. Conway, R. E. Verall, and J. E. Desnoyers, Z. Physik. Chem. (Leipzig) 230, 157 (1965)

${ }_{13} \mathrm{~J}$. E. Desnoyers and G. Jolicoeur, in Modern Aspects of Electrochemistry, edited by J. O'M. Bockris and B. E. Conway (Plenum, New York, 1969), Vol. 5, p. 1.

14 J. Padova, J. Chem. Phys. 40, 691 (1964).

${ }_{15} \mathrm{H}$. S. Harned and B. B. Owen, The Physical Chemistry of Electrolyle Solutions (Reinhold, New York, 1958), 3rd ed., p. $79,80$.

i6 J. E. B. Randles, Trans. Faraday Soc. 52, 1573 (1956).

17 F. O. Koenig, CITCE, 3rd Reunion, Manfredi, Milan (1952), p. 299.

${ }_{18}$ B. B. Owen, J. Am. Chem. Soc. 54, 1758 (1952).

${ }^{19}$ For a recent review, cf. O. Popovych, Crit. Rev. Anal. Chem. $1,43(1970)$.

THE JOURNAL OF CHEMICAL PHYSICS

\title{
Stability of Gas Bubbles in a Deformable Material Containing Dissolved Gases
}

\author{
WEN-JEt Yang \\ Department of Mechanical Engineering, The University of Michigan, Ann Arbor, Michigan 48104
}

(Received 4 December 1970)

\begin{abstract}
This paper analyzes the stability of gas bubbles in a medium which contains dissolved gases and deforms due to creep. The effects of mass diffusion and surface tension on the dynamic behavior of a gas bubble are taken into account. Asymptotic solutions valid for large times are obtained which predict the radius-time relation, the creep rate, and the rate of diffusion of dissolved gases induced by the growth of a gas bubble in an oversaturated solution. The case where the bubble is situated in a saturated or undersaturated solution is also examined.
\end{abstract}

\section{INTRODUCTION}

Considerable attention has recently been focused on the dynamics of a gas or vapor bubble in a boiling liquid, in a liquid-gas solution, or in a cavitating flow of a liquid. A vapor bubble in a boiling liquid will grow or shrink by heat diffusion according as the liquid is superheated, saturated, or subcooled. Analogously, the growth or collapse of a gas bubble in a liquid-gas solution will occur according as the solution is oversaturated or undersaturated. In a cavitating flow of a liquid, however, the growth or collapse of a gas bubble is controlled by the external pressure, surface-tension force, viscous force, and inertia forces.

In the present paper, the problem dealing with the dynamic behavior of a gas bubble situated in a deformable medium containing dissolved gases is formulated. The effects of mass diffusion and surface tension on bubble dynamics are taken into consideration. Approximate solutions valid for large times are obtained through which stability of a gas bubble in an oversaturated, saturated, or undersaturated solution is examined.

Results of the study may be applied to the swelling of fissionable materials caused by the growth of inert gas bubbles which are generated as a result of irradiation, ${ }^{1-8}$ the expansion or shrinkage of gas emboli formed in extravascular tissues or organs, ${ }^{9,10}$ the growth or collapse of gas bubbles in polymer melts during plastic foaming process, etc.

\section{ANALYSIS}

Consider a spherical gas bubble situated in an infinite region of a homogeneous material in which the distribution of the stresses is spherically symmetrical around the bubble. At the initial time $t=0$, the radius of the bubble is $R_{0}$ and the concentration of the gas dissolved in the material is uniform and equal to $C_{0}$. The system is assumed to be at constant temperature $T$ and pressure, and the equilibrium concentration of the dissolved gas at this temperature and pressure is $C_{s}$. The center of the gas bubble is taken as the origin of a spherical polar coordinate system $(r, \theta, \phi)$.

Owing to spherical symmetry, the nondiagonal components $\tau_{\theta \phi}, \tau_{\theta r}$, and $\tau_{\phi r}$ of the stress tensor are equal to zero and $\tau_{\theta \theta}=\tau_{\phi \phi}=-\tau_{r r} / 2$. In order to describe the process of creep in the material, it is proposed that the creep rate $\epsilon_{\tau r}=d v_{r} / d r$ may be related to the intensity of the tangential stresses $\tau_{\theta \theta}-\tau_{r r}$ by the expression

$$
\epsilon_{r r}=-\left(\tau_{\theta \theta}-\tau_{r r}\right)^{a} / b,
$$

where $v_{r}$ is the radial component of the deformation rate $\mathbf{v}$ and $a$ and $b$ are nonzero constants determined by the properties of the material, the temperature, etc. 
However, it is required that

$$
\nabla \cdot \nabla=\left(d v_{r} / d r\right)+\left(2 v_{r} / r\right)=0 .
$$

Hence, $v_{r} r^{2}$ must be a function of time only or at any time $t>0$

$$
v_{r}=(R / r)^{2} d R / d t
$$

where $R$ is the instantaneous radius of the bubble. The stresses $\tau_{\mathrm{rr}}$ and $\tau_{\theta \theta}$ must satisfy the equilibrium equation

$$
\left(d \tau_{r r} / d r\right)+(2 / r)\left(\tau_{r r}-\tau_{\theta \theta}\right)=0
$$

subject to the boundary conditions

$$
\begin{array}{ll}
r=R: & -\tau_{r r}+(2 \sigma / R)=P_{\theta}, \\
r=\infty: & \tau_{r r}=0,
\end{array}
$$

in which $\sigma$ is the surface tension and $P_{g}(t)$ is the gas pressure inside the bubble. Equation (5) signifies the balance of forces at the bubble surface.

With the substitution of Eqs. (1) and (3), Eq. (4) is solved with the boundary conditions (5) and (6). It yields

$$
d R / d t=(R / 2 b)(3 / 2 a)^{a}\left[P_{g}-(2 \sigma / R)\right]^{a} .
$$

If the gas in the bubble behaves like an ideal gas, the equation of state reads

$$
P_{g}(4 \pi / 3) R^{3}=m(t) \bar{R} T,
$$

wherein $m(t)$ is the mass of the gas contained in the bubble and $\bar{R}$ is the gas constant. Now, Eqs. (7) and (8) are combined to eliminate $P_{0}$. It gives

$$
\left(\frac{d R^{3 a}}{d t}\right)^{1 / a}=\left(\frac{3 a}{2 b}\right)^{1 / a}\left(\frac{3}{2 a}\right)\left(\frac{3 m \bar{R} T}{4 \pi}+2 \sigma R^{2}\right) .
$$

The differentiation of Eq. (9) with respect time produces

$\frac{d}{d t}\left(\frac{d R^{3 a}}{d t}\right)^{1 / a}=\left(\frac{3 a}{2 b}\right)^{1 / a}\left(\frac{3}{2 a}\right)\left(\frac{3 \bar{R} T}{4 \pi} \frac{d m}{d t}+4 \sigma R \frac{d R}{d t}\right)$.

Next, the diffusion of the dissolved gas to or from the bubble will be investigated. At any time $t>0$ when the bubble radius is $R$, the dissolved gas concentration $C(r, t)$ at a point in the material at a distance $r$ from the origin is to be found from the mass diffusion equation which reads

$$
\partial C / \partial t=D \nabla^{2} C,
$$

where $D$ is the diffusion coefficient of the gas in the material. The appropriate initial and boundary conditions are

$$
\begin{gathered}
C(r, 0)=C_{0}, \quad \text { for } r>R \\
C(R, t)=C_{s}, \quad C(\infty, t)=C_{0}, \quad \text { for } t>0 .
\end{gathered}
$$

Through the transformation using $u=r\left(C-C_{s}\right)$ as the dependent variable and a linear shift in the $r$ coordi- nate by $\xi=r-R$, the solution can be easily found to be

$$
\begin{aligned}
C(r, t)=C_{8}+\frac{\left(C_{0}-C_{s}\right)}{2 r(\pi D t)^{1 / 2}} \int_{0}^{\infty}\left(R+\xi^{\prime}\right)\left[\exp \left(\frac{-\left(\xi-\xi^{\prime}\right)^{2}}{4 D t}\right)\right. \\
\left.-\exp \left(\frac{-\left(\xi-\xi^{\prime}\right)^{2}}{4 D t}\right)\right] d \xi^{\prime} . \quad
\end{aligned}
$$

The concentration gradient at the bubble surface is found directly from Eq. (14) as

$$
(\partial C / \partial r)_{r=R}=\left(C_{0}-C_{s}\right)\left[R^{-1}+(\pi D t)^{-1 / 2}\right] .
$$

Since the rate of the dissolved gas flowing into or out of the bubbles is

$$
d m / d t=4 \pi R^{2} D(\partial C / \partial r)_{r=R}
$$

therefore the combination of Eqs. (10), (15), and (16) yields

$$
\begin{aligned}
& \frac{d}{d t}\left(\frac{d R^{3 a}}{d t}\right)^{1 / a}=\left(\frac{3 a}{2 b}\right)^{1 / a}\left(\frac{3}{2 a}\right) \\
& \quad \times\left[3 \bar{R} T R^{2} D\left(C_{0}-C_{s}\right)\left[R^{-1}+(\pi D t)^{-1 / 2}\right]+4 \sigma R \frac{d R}{d t}\right]
\end{aligned}
$$

One can rewrite Eq. (17) in dimensionless form as

$$
\begin{aligned}
\frac{d}{d \tau}\left(\frac{d \rho^{3 a}}{d \tau}\right)^{1 / a} & =\left(\frac{3}{2} a\right)^{1 / a}\left(\frac{3}{2 a}\right) \\
\times \Phi & {\left[3 \rho^{2}\left(1-X_{s}\right)\left(\rho^{-1}+(\pi \tau)^{-1 / 2}\right)+4 \gamma \rho \frac{d \rho}{d t}\right] }
\end{aligned}
$$

in which

$$
\begin{gathered}
\rho=R / R_{0}, \quad \tau=D t / R_{0}{ }^{2}, \quad \Phi=\bar{R} T C_{0} /\left(\frac{b D}{R_{0}^{2}}\right)^{1 / a}, \\
X_{s}=C_{s} / C_{0}, \quad \gamma=\sigma /\left(R_{0} C_{0} \bar{R} T\right) .
\end{gathered}
$$

The initial conditions are $R=R_{0}$ and $d R / d t=0$ or

$$
\tau=0: \quad \rho=1, \quad d \rho / d \tau=0 .
$$

Thus, the problem of determining the dynamic behavior of a gas bubble or the time history of the deformation rate is now reduced to solving the nonlinear Eq. (18) subject to the appropriate initial conditions (20). Unfortunately, it is impossible to solve this analytically over the entire time domain. Numerical solutions can be easily obtained by the Runge-Kutta method with the aid of a digital computer. However, from the viewpoint of pratical interest in the large-time behavior, effort will be directed toward finding the asymptotic solution of Eq. (18) in the following.

When enough time has elapsed, the term $1 /(\pi \tau)^{1 / 2}$ in Eq. (18) becomes much less than $1 / \rho$. In the absence of the surface-tension effect, the asymptotic solution [satisfying Eq. (20) exactly and Eq. (18) 
approximately] is ${ }^{11}$

where

$$
\rho=1+(A \tau)^{(a+1) / 2 a},
$$

$A=\left\{[a /(a+1)]^{1 / a}[9 \Phi /(3 a+1)]\left(1-X_{s}\right)\right\}^{a /(a+1)}$.

When the material is oversaturated with the dissolved gas, i.e., $X_{s}<1$, it is evident from Eq. (21) that a gas bubble will grow. On the other hand, $\left(1-X_{s}\right)^{a /(a+1)}$ becomes a complex number for $X_{s}>1$ indicating that no gas bubble can ever exist in an undersaturated solution.

The radial component of the deformation rate in the material is, from $\mathrm{Eq}$. (3),

$i_{r}^{\prime}=\left(R_{0} D / r^{2}\right)[(a+1) / a] A(A \tau)^{(1-a) / 2 a}\left[1+(A \tau)^{(a+1 / 2 a}\right]^{2}$.

The rate of mass diffusion into the bubble, Eq. (16), can be written in dimensionless form as

$d M / d \tau=3 \rho\left(1-X_{s}\right)=3\left(1-X_{s}\right)\left[1+(A \tau)^{(a+1) / 2 a}\right]$,

where $M$ is defined as $m / m_{0}$ with $m_{0}=4 \pi R_{0}{ }^{3} C_{0} / 3$.

Several special cases are of particular interest:

(i) In a saturated solution, $X_{s}=1$ : Equation reduces to

$$
\frac{d}{d \tau}\left(\frac{d \rho^{3 a}}{d \tau}\right)^{1 / a}=\left(\frac{3}{2} a\right)^{1 / a}\left(\frac{3 \Phi \gamma}{a}\right) \frac{d \rho^{2}}{d \tau} .
$$

Or in a more tractable form

$$
d \rho^{3 a} / d \tau=\left(\frac{3}{2} a\right)(3 \Phi \gamma / a)^{a}\left(\rho^{2}-1\right) .
$$

Its solution is

$$
\begin{aligned}
\rho^{3 a} & \sum_{n=1}^{\infty} \frac{a(a+1)(a+2) \cdots(a+n-1) \rho^{2 n}}{n !(3 a+2 n)} \\
& -\sum_{n=1}^{\infty} \frac{a(a+1)(a+2) \cdots(a+n-1)}{n !(3 a+2 n)}=-\frac{1}{2} \tau\left(\frac{3 \Phi \gamma}{a}\right)^{a} .
\end{aligned}
$$

At large times when $\rho^{2}$ becomes negligible in comparison with unity, the asymptotic solution of $\mathrm{Eq}$. (25) is

$$
\rho=\left[1-(3 a / 2)\left(3 \Phi_{\gamma} / a\right)^{a} \tau\right]^{3 a} .
$$

If surface tension is neglected, a bubble of any radius would be stable against diffusion in a saturated solid solution.

(ii) $b \rightarrow \infty$ or $\epsilon_{r r} \rightarrow 0$ : When the creep rate is very small, a gas bubble will grow or shrink by diffusion alone. For the mass diffusion-controlled case, it can be shown that its dynamic behavior is governed by the equation

$$
\frac{d \rho}{d \tau}=\frac{1-X_{s}}{X_{\rho}+2 \gamma / 3 \rho}\left[\rho^{-1}+(\pi \tau)^{-1 / 2}\right],
$$

where $X_{\rho}=d_{g} / C_{0}$ and $d_{g}$ is the gas density under the same conditions of pressure and temperature with an interface of zero curvature. Equation (28) shows that a gas bubble will grow or collapse according as the solution is oversaturated or undersaturated. For a saturated solution, $X_{s}=1$, a bubble would be stable only in the absence of surface tension. The asymptotic solution of Eq. (28) for large times is

$\rho=(1 \pm \tau)^{1 / 2}\left\{\begin{array}{l}+ \text { for an oversaturated solution } \\ - \text { for an undersaturated solution }\end{array}\right.$

in the absence of surface tension. Details on the stability of gas bubbles in liquid-gas solutions due to diffusion are given in Ref. 12.

(iii) $a=1$ and $b=-3 \mu$ : This corresponds to a viscous fluid in which $\mu$ represents its absolute viscosity. Equations (21) and (22) indicates that a bubble will grow or shrink as a linear function of time for the case where creep deformation controls. On the other hand, growth or collapse is a square-root function of time for the diffusion-controlled case as shown by Eq. (29).

\section{CONCLUSIONS}

A gas bubble in a deformable medium oversaturated with dissolved gases will grow, while that in an undersaturated medium cannot exist. In a saturated medium, however, a gas bubble will shrink due to surface tension. At large times, the bubble radius, the creep rate, and the rate of diffusion of dissolved gas all vary with power functions of time. Special cases reduce to the problems of the diffusion-controlled bubble dynamics and the dynamics of a gas bubble in viscous liquids.

${ }^{1}$ A. J. Markworth, J. Appl. Phys. 40, 1987 (1969).

2 E. M. Baroody, J. Appl. Phys. 38, 4893 (1967).

${ }^{3}$ V. V. Slezov and V. B. Shikin, EuroNuclear 2, 127 (1965)

${ }^{4}$ L. P. Semenov, Sov. J. At. Energy English Transl. 15, 1155 (1963).

${ }^{5}$ V. M. Agranovich, E. Ya Mikhlin, and L. P. Semenov, Sov. J. At. Energy English Transl. 15, 1140 (1963).

${ }_{6}^{6}$ V. M. Agranovich and E. Ya. Mikhlin, Sov. J. At. Energy English Transl. 12, 410 (1962).

${ }^{7}$ G. W. Greenwood and A. Boltax, J. Nucl. Mater. 5, 234 (1962).

${ }^{8}$ S. F. Pugh, J. Nucl. Mater. 4, 177 (1961).

9 K. S. Chan and W. J. Yang, J. Biomechanics 2, 299 (1969).

${ }^{10}$ E. N. Harvey, D. K. Barnes, W. D. McElory, A. H. Whiteley, D. C. Pease, and K. W. Cooper, J. Cellular Comp. Physiol. 24, 1 (1944).

"In addition to satisfying the governing equation (18), $\rho=(1+A \tau)^{(a+1) / 2 a}$ and $\rho=(A \tau)^{(a+1) / 2 a}$ satisfy the initial conditions $\rho(0)=1$ and $d \rho(0) / d \tau=0$, respectively.

12 P. S. Epstein and M. S. Plesset, J. Chem. Phys. 18, 1505 (1950). 